\title{
A rare case of intramural patent ductus arteriosus in a dog: anatomic, histopathologic, and radiographic features
}

\author{
Jung-Hyun Kim¹, Su-Min Kim², Ja-Young Kim³, Hyun-Jung Han ${ }^{3, *}$ \\ ${ }^{1}$ Department of Veterinary Internal Medicine, College of Veterinary Medicine, Konkuk University, Seoul, Korea \\ ${ }^{2}$ Department of Veterinary Internal Medicine and ${ }^{3}$ Department of Veterinary Emergency, \\ Konkuk University Veterinary Medical Teaching Hospital, Seoul, Korea
}

\begin{abstract}
A 5-year-old dog was evaluated for a lethargy and respiratory distress. Radiograph revealed cardiomegaly with pleural effusion, and the dog died during a thoracocentesis. At necropsy, the descending aorta and pulmonary trunk were dilated and in close contact, but there was no external evidence of a patent ductus arteriosus (PDA). When the descending aorta was opened however, an ostium opening into the pulmonary trunk was evident. Histopathological investigation revealed that the intramural PDA resembled vascular tissue with a structure and architecture. The diagnosis was an intramural PDA, an extremely rarely reported type of PDA in the dog.
\end{abstract}

Keywords: canine, congenital heart disease, intraaortic shelf, pleural effusion

\section{*Corresponding author}

Hyun-Jung Han

Department of Veterinary Emergency, Konkuk University Veterinary Medical Teaching Hospital, 120 Neungdong-ro, Gwangjin-gu, Seoul 05029, Korea

Tel: $+82-2-450-3664$

Fax: +82-2-444-4396

E-mail: ab1234@konkuk.ac.kr

ORCID

Jung-Hyun Kim

https://orcid.org/0000-0003-0719-3266

Su-Min Kim

https://orcid.org/0000-0003-3107-3133

Ja-Young Kim

https://orcid.org/0000-0001-9818-5709

Hyun-Jung Han

https://orcid.org/0000-0002-1910-1261

Conflict of Interest

The authors declare no conflicts of interest.

Received: February 13, 2020

Revised: April 02, 2020

Accepted: April 07, 2020
Patent ductus arteriosus (PDA) is one of the most common congenital heart diseases in dogs $[1,2]$. PDA is characterized by the persistence of the ductus arteriosus, which shunts blood from the pulmonary artery to the aorta during fetal development and generally closes by 7 or 8 days post whelping, with histologic studies suggesting even earlier closure [3,4]. When the ductus fails to close after birth, the rise in systemic arterial pressure and the fall in pulmonary arterial pressure usually results in a left-to-right shunt in which blood flows from the aorta to the pulmonary artery [3].

Standard morphologies of PDA that have been reported include extramural PDAs have been angiographically classified into either four [5] or five [6] phenotypes. Intramural PDA has not been included in any of the previously reported classification systems, and only one case has been reported to date [7]. In that case, the PDA was contained entirely within the wall of the aorta, and the intramural aspect of the PDA was not evident on echocardiography [7]. When surgical correction was attempted in that case, ligation was not possible due to the absence of a discrete ductal PDA vessel, and that dog died from cardiac arrest during surgery. Herein, we report the fatal clinical course of a dog with intramural PDA, including details pertaining to the clinical, gross anatomic, and histopathological findings. To our knowledge, this is the first report of the radiographic and histopathological features of canine intramural PDA in the literature. Our description of the precise anatomical structure of this extremely rare congenital heart disease may help clinicians diagnose and manage intramural PDA in the future.

A 5-year-old, 2.8-kg female Maltese dog was referred to the Veterinary Medical Teaching Hospital for evaluation of a 3-day history of acute lethargy and respiratory distress. The owner reported that the dog had been less active since the patient was a puppy, however the respiratory distress was firstly found one month ago and severely worsened 3 days ago. When the dog was examined by the referring veterinarian earlier at the presentation day, it showed profound respiratory distress. Although the referring veterinarian diagnosed the cardiomegaly suspected pericardial effusion based on radiographic examination, any medication was not prescribed. The dog was referred to our hospital for further diagnostic evaluation and management. On 


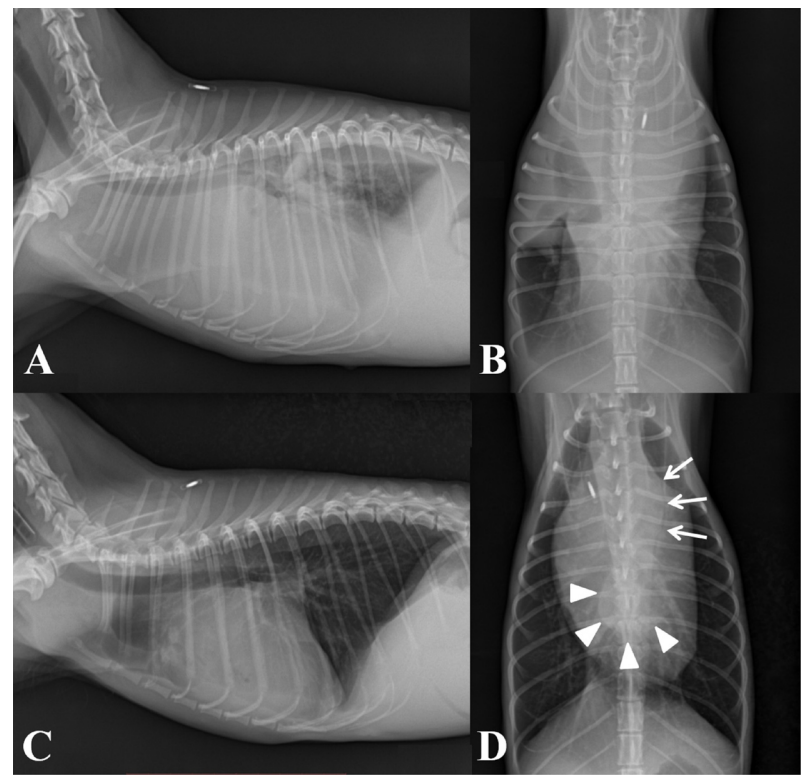

Fig. 1. Thoracic radiograph of a dog with intramural patent ductus arteriosus before (A, B) and after (C, D) thoracocentesis. (A) On the lateral view, radiopaque fluid obscures much of the thoracic detail and the trachea is elevated dorsally. (B) On the ventrodorsal view, the radiolucent lung regions are markedly retracted from the thoracic wall, especially the cranial lung lobe region. (C) After thoracocentesis, cardiomegaly is detected with bulging of the cardiac silhouette at 2 o'clock on the lateral view. (D) On the ventrodorsal view, the leftward bulge of the descending aorta (arrows) and a round radiopaque structure (arrowheads) are seen, mostly likely representing the left atrium superimposed over the cardiac silhouette. Marked elongation of the heart is also evident.

physical examination, the femoral pulses were weak and mucous membranes were pale. The heart rate was 120 beats per minute, and the heart and breath sounds were muffled. Thoracic radiography revealed complete obliteration of the cardiac silhouette with tracheal elevation on the lateral view and were consistent with pleural effusion and cardiomegaly (Fig. 1A). On the ventrodorsal view, scalloping of the right cranial and middle lung margins and interlobar fissure lines were visible (Fig. 1B). On abdominal radiography, peritoneal effusion and hepatomegaly were also detected. Base on the radiographic findings, a differential diagnosis included pure pleural effusion, pyothorax, hemothorax, bivalvular insufficiency, pericardial effusion, pulmonary hypertension, and reversed PDA. Supplemental oxygen (inspired $\mathrm{O}_{2}$ fraction $40-60 \%$ ) was provided via an oxygen cage and prompt thoracocentesis was attempted; however, the dog rapidly deteriorated and cardiac arrest occurred. Despite prompt cardiopulmonary resuscitation with cardiac massage and the administration of anticholinergic (atropine $0.05 \mathrm{mg} / \mathrm{kg} \mathrm{IV}$; Jeil atropine sulfate injection $0.5 \mathrm{mg} / \mathrm{mL}$, Jeil pharmaceutical, Korea) and sympathomimetic (epinephrine 0.01-0.1 mg/ kg IV; Daihan epinephrine injection $1 \mathrm{mg} / \mathrm{mL}$, Daihan Pharm.
Co., LTD, Korea) agents, the dog died. With the owner's consent, the dog's body was donated to the hospital for further study. Before necropsy, experimental thoracocentesis was performed within 30 minutes after death, and a total of $270 \mathrm{~mL}$ of pleural effusion was removed. The pleural effusion was diagnosed as transudative and cytology of the pleural effusion revealed no remarkable findings. After the thoracocentesis, thoracic radiography revealed generalized cardiomegaly (vertebral heart score 11.8), bulging of the descending aorta, and enlargement of the left atrium (Fig. 1C, 1D).

At necropsy, mild to moderate pleural effusion was identified, and the lungs were diffusely congested with foamy blood present in the trachea and pulmonary parenchyma, which was consistent with pulmonary hemorrhage likely secondary to cardiopulmonary resuscitation. Additionally, severe cardiomegaly with eccentric right ventricular hypertrophy was identified, and the main pulmonary veins and arteries were dilated. The descending aorta and pulmonary trunk were also dilated and in close contact, but there was no external evidence of a vessel connecting the aorta and pulmonary artery. When the descending aorta was opened however, a 3$\mathrm{mm}$ ostium opening into the pulmonary artery was evident. Further distal dissection of the aorta revealed the presence of a 1-cm long intraaortic shelf that divided the intramural PDA and descending aorta (Fig. 2). Additionally, moderate ascites and hepatomegaly were identified. A necropsy sample of the intramural PDA was fixed via immersion in neutral-buffered $10 \%$ formalin for microscopic evaluation. Histopathological examination of the specimen revealed that it comprised a variably thick layer of smooth muscle tunic with small amounts of fibrous tissue (Fig. 3). There were thin lining of endothelium and scant mucin-laden connective tissue stroma. Focal areas of mural mineralization and some subintimal mucin accumulation were noted. No inflammation was apparent. These features of the multifocal areas, including mild mineralization, muscular tunic, and tunica media, were indicative of an aortic vessel. Based on the gross anatomical and histopathological findings, the dog was ultimately diagnosed with intramural PDA.

Until recently, reports of PDA in dogs have been limited to extramural cases [5]. Das et al. [7] reported the first case of canine intramural PDA. In that report, intramural PDA was diagnosed postmortem. Because intramural PDA is evidently difficult to identify without necropsy, its true prevalence is unknown.

The common radiographic findings in previously reported cases of extramural left-to-right shunting PDA include progressive enlargement of the left atrium, left ventricle, aortic arch, and pulmonary arteries $[1,8]$. Pulmonary overcirculation can result in dilation of the pulmonary arteries and veins, with vessel diameters correlating with the shunt volume [3]. Often, an aneurysmal bulge is also evident in the descending aorta at the level of the ductus arteriosus [3]. Although aneurysmal bulge of the aorta is most readily detected in dorso- 


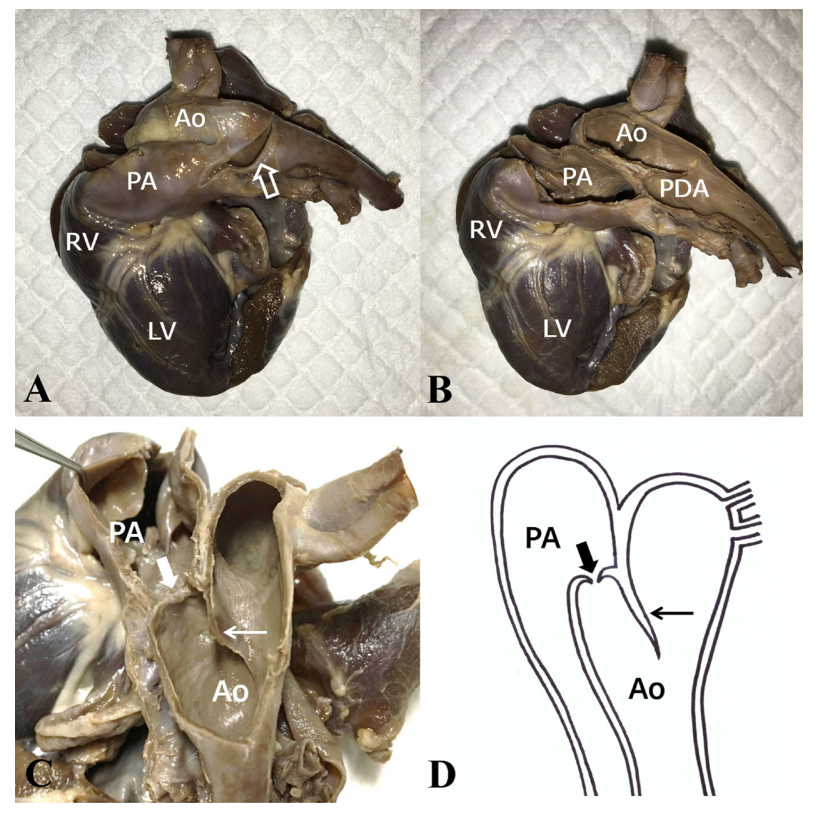

Fig. 2. Necropsy of the heart in a dog with intramural PDA. (A) The descending aorta and pulmonary trunk are dilated. The dissection line of the ligamentum arteriosus is noted (open arrow). $(\mathrm{B}, \mathrm{C})$ Further dissection of the pulmonary artery and aorta reveals intramural PDA within the aorta. The intramural PDA starts from the orifice connecting the pulmonary artery to the aorta along the intraaortic shelf. (D) Diagram illustrating the ostium (thick arrow), and intraaortic shelf (thin arrow) in the same dog. Ao, aorta; LV, left ventricle; RV, right ventricle; PA, pulmonary artery; PDA, patent ductus arteriosus.

ventral radiographs, where it causes a lateral deviation of the left lateral wall of the descending aorta at the level of the main pulmonary artery [3], it may also be seen in ventrodorsal radiography when it is significant. The progression of PDA is associated with excess pulmonary blood flow, which induces pulmonary vascular changes such as increased pulmonary vascular resistance and pulmonary hypertension [3]. Additionally, right heart enlargement may be a sign of advanced heart failure [1]; the present case also demonstrated right ventricular dilation. Lastly, if pulmonary arterial pressure exceeds aortic pressure, blood flow through the PDA can be reversed (right-to-left shunt) in some cases [1,5]. Reversed shunting can induce secondary pulmonary hypertension and right congestive heart failure, which can manifest as pleural effusion or ascites on radiographic examination, both of which were evident in the current case. Unfortunately, although the pulmonary hypertension was not evaluated by echocardiographically in the present case, reverse shunting PDA was suspected because presence of marked pleural and peritoneal effusion, and grossly identified eccentric right ventricular hypertrophy during necropsy. Furthermore, as seen in the present case, canine hearts with PDA often exhibit an elongated appearance because the left ventricular enlargement extends the cardiac silhouette caudally

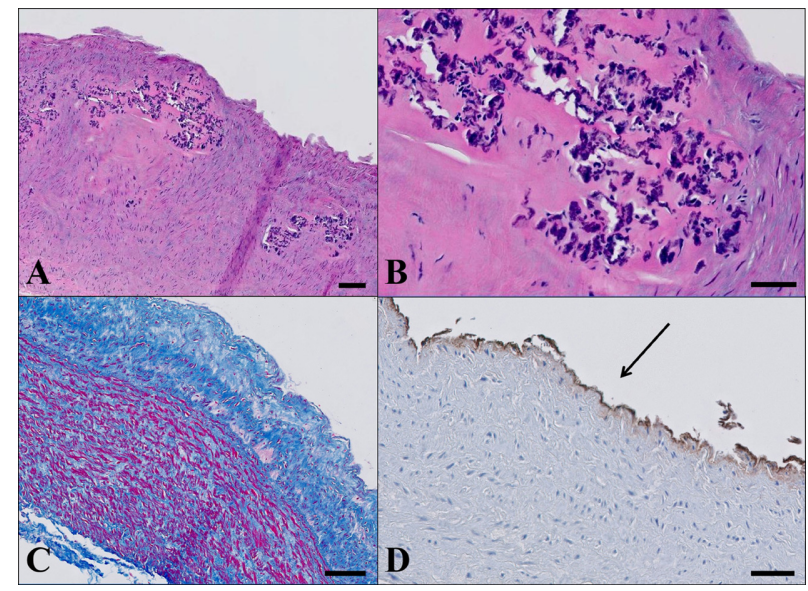

Fig. 3. Histopathology of the intramural patent ductus arteriosus. (A) This structure comprises a variably thick layer of smooth muscle tunic with small amounts of fibrous tissue. $\mathrm{H} \& \mathrm{E}, \mathrm{Bar}=100 \mu \mathrm{m}$. (B) Focal areas of mural mineralization and some subintimal mucin accumulation are seen. H\&E, Bar = $50 \mu \mathrm{m}$. (C) Masson trichrome straining demonstrates a normal component of collagenous connective tissue (blue color) in the vessel wall, as well as smooth muscle (red). Bar $=100 \mu \mathrm{m}$. (D) Factor VIII immunohistochemical staining demonstrates a thin lining of endothelium (arrow) along the lumen of the aorta. Bar $=50 \mu \mathrm{m} . \mathrm{H} \& \mathrm{E}$, hematoxylin and eosin.

while the dilated aortic arch extends the cardiac silhouette cranially [9]. In a previous case report [7], intramural PDA could not be differentiated from extramural PDA via echocardiography. Anatomical evaluation via necropsy, as was utilized in the present case, could help diagnose intramural PDA.

In the present case, histopathological investigation revealed that the intraaortic shelf of the intramural PDA resembled vascular tissue with a structure and architecture that was very similar to the aorta, comprising a variably thick tunica intima and tunica media with areas of mineralization. There was no discrete separation or membranous type tissue visible microscopically. The mineralization was identified primarily within the tissue of the ostium, but the architecture at each site appeared to be otherwise similar. The cause of the mineralization is unclear. Vascular mineralization is occasionally seen in hypothyroidism canines that develop atherosclerosis [10]; however, there was no lipid accumulation or any other evidence of atherosclerotic plaque or cholesterol cleft formation in the present case. The foci consisted of pure mineralization of the smooth muscle stromal tissue with no evidence of fatty deposits or secondary inflammation. This mineralization may be found incidentally in some patients, but it may also precede dissecting aneurysm [11]. Because the anatomical structure of the intraaortic shelf of the intramural PDA was very close to that of the aorta, it may have been some type of diverticulum or aneurysm arising from the aorta or a developmental lesion of some kind. 
Dogs with PDA rarely survive to a normal age due to the development of congestive heart failure [3]. Therefore, when left-to-right shunting PDA is diagnosed, closure via surgical ligation or interventional occlusion is usually recommended [3]. Generally, the only contraindication for the surgical correction of left-to-right shunting PDA has been the presence of a coexisting cyanotic cardiac abnormality such as tetralogy of Fallot [3]. In a previous report [7], however, an intramural PDA was reported to be inoperable because it was contained entirely within the aortic wall and there was no surgically accessible segment. In other words, it was an intraaortic shelf rather than a duct. Although there have been no reports of interventional correction of intramural PDA in dogs, transvenous or transarterial coil embolization or the use of an Amplatz canine ductal occluder could be considered after confirmation of left-to-right shunting PDA in a patient who has an intraaortic shelf separating the ductus from the descending aorta. However, the right to left shunting PDA, as was suspected in this case, is a contraindication to PDA closure.

This is the first description of radiographic and histopathologic features with necropsy of intramural PDA in a dog. We believe that this case is important because the extraordinary clinical features, radiographic, necropsy, and histopathologic findings of canine intramural PDA have only been reported extremely rarely. In conclusion, intramural PDA should be considered in the differential diagnosis of congenital heart disease inducing cardiomegaly and pleural effusion in dogs. Additionally, when canine PDA is suspected, it is important that veterinarians recognize the rarely occurring intramural PDA. Furthermore, although there has not been reported yet, advanced imaging including computed tomography angiography or magnetic resonance angiography, especially $3 \mathrm{D}$ or $4 \mathrm{D}$ modalities available, could be theoretically considered to improve diagnostic accuracy for this defect.

\section{References}

1. Doocy KR, Saunders AB, Gordon SG, Jeffery N. Comparative, multidimensional imaging of patent ductus arteriosus and a proposed update to the morphology classification system for dogs. J Vet Intern Med 2018;32:648-657.

2. Schrope DP. Prevalence of congenital heart disease in 76,301 mixed-breed dogs and 57,025 mixed-breed cats. J Vet Cardiol 2015;17:192-202.

3. Buchanan JW. Patent ductus arteriousus morphology, pathogenesis, types and treatment. J Vet Cardiol 2001;3:7-16.

4. Fox PR, Sisson D, Moise NS. Textbook of canine and feline cardiology: principles and clinical practice. 2nd ed. p. 505, WB Saunders, Philadelphia, 1999.

5. Miller MW, Gordon SG, Saunders AB, Arsenault WG, Meurs KM, Lehmkuhl LB, Bonagura JD, Fox PR. Angiographic classification of patent ductus arteriosus morphology in the dog. J Vet Cardiol 2006;8:109-114.

6. Schneider M, Schneider I, Hildebrandt N, Wehner M. Percutaneous angiography of Patent Ductus Arteriosus in dogs: techniques, results and implications for intravascular occlusion. J Vet Cardiol 2003;5:21-27.

7. Das S, Yool DA, French AT, Self IA, del-Pozo J. An unusual morphology of patent ductus arteriosus in a dog. $\mathrm{J}$ Small Anim Pract 2012;53:353-356.

8. Lee Y, Jung J, Park J, Jeong J, Jeon S, Park S, Chang J, Kang JH, Lee C, Chang D. Cardiac magnetic resonance imaging of patent ductus arteriosus in three dogs. Vet Radiol Ultrasound 2017;58:62-75.

9. Broaddus K, Tillson M. Patent ductus arteriosus in dogs. Compend Contin Educ Vet 2010;32:E3.

10. Schwarz T, Sullivan M, Störk CK, Willis R, Harley R, Mellor DJ. Aortic and cardiac mineralization in the dog. Vet Radiol Ultrasound 2002;43:419-427.

11. Barrett HE, Cunnane EM, O Brien JM, Moloney MA, Kavanagh EG, Walsh MT. On the effect of computed tomography resolution to distinguish between abdominal aortic aneurysm wall tissue and calcification: a proof of concept. Eur J Radiol 2017;95:370-377. 